\title{
DEVELOPMENT OF CARBOXYMETHYL CELLULOSE/ POLYPHENOLS GELS FOR TEXTILE APPLICATIONS
}

\author{
Hana Krizova, Jakub Wiener \\ Technical University of Liberec, Faculty of Textile Engineering, Studentska 2, Liberec 46117, Czech Republic \\ E-mail: hana.krizova@tul.cz, jakub.wiener@tul.cz
}

\begin{abstract}
:
The aim of this study was to determine release rate and changes in polyphenols' content, which were sorbed to carboxymethyl cellulose gel and subsequently desorbed. An aqueous extract of blue marc vine variety Fratava was used as a source of polyphenols. The gel was dried into a solid film and polyphenols were then desorbed again by dissolving this film in saline (isotonic) solution. Further, the influence of different times of high temperature $\left(180^{\circ} \mathrm{C}\right)$ of drying gel on change in the amount of released polyphenols and also kinetics of their release in re-transfer of the film on the gel and solution was studied. The process simulates the possible use of carboxymethyl cellulose/ polyphenols film sorbed on textile materials and its contact with the tissues and body fluids such as course of wound healing.
\end{abstract}

\section{Keywords:}

Carboxymethyl cellulose, polyphenols, desorption, thermal crosslinking

\section{Introduction}

\subsection{Polyphenols (PF)}

Polyphenols are a very varied group of chemically diverse compounds that contain hydroxyl groups bound to the aromatic ring. They are easily oxidizable substances with a low redox potential which are able to reduce some radicals (e.g. superoxide, peroxyl and hydroxyl ones) with oxidative effects. Polyphenols are a broad group of plant bioactive substances able to protect cells from oxidative damage due to their strong antioxidant and antiradical activities. PF include for example amino acids (tyrosine), essential oils, phenolic acids (e.g. vanil, gallic, coumaric and ferulic ones), flavonoids (e.g. catechins, quercetin, rutin, anthocyanidins) and tannins.

\section{$\underline{1.2 \text { Tannins }}$}

Tannins are oligomeric and polymeric polyphenol compounds contained mainly in the leaves and bark of trees, but also in seeds, herbal stems, tea, oak apples, fruits, vegetables and vine (especially in the red one). Tannins are used to protect plants against pests, parasites and adverse conditions. They are substances with a wide spectrum of biogenic effects. Often it is a bitter substance and adstringens (drugs with astringent effect) inhibiting glandular secretion, act local vasoconstriction and has antidiarrheal effect. Tannins form through their hydroxyl and carboxyl groups complexes with various ingredients especially with proteins (coagulation proteins which are based on the process of tanning of skin tannins), amino acids and alkaloids. They form complexes with metal ions, carbohydrates and fats. These chemical properties also lead to antimicrobial effects of tannins as complexation of enzymes and ions may subsequently inhibit proliferation of microbes and some molds. Tannins are generally divided into two large groups.

\subsubsection{Hydrolyzable tannins}

Their basic monomer unit is gallic or ellagic acid and their molecular weight ranges from 500 to 3000 . They are well soluble in water, hydrolyze due to heat, weak acids or weak bases. They are also easily decomposed by digestive enzymes of mammals. Among these is e.g. oenothein present in the wine or tannic (Figure 1) [1] contained in fruits and bark of oak, chestnut or in leaves of sicilian sumac [1].

\subsubsection{Condensed tannins (proanthocyanidins)}

Their basic unit of condensed tannins is a monomeric flavan-3-ol (Figure 2) [1], and according to the degree of polymerization their molecular weight also reaches over 20,000. Condensed tannins contain strong bonds between carbons, and are therefore not easily hydrolyzable and in the tract of mammals hardly decompostable. An example of condensed tannins is procyanidin or prodelphinidin. Condensed tannins accumulate

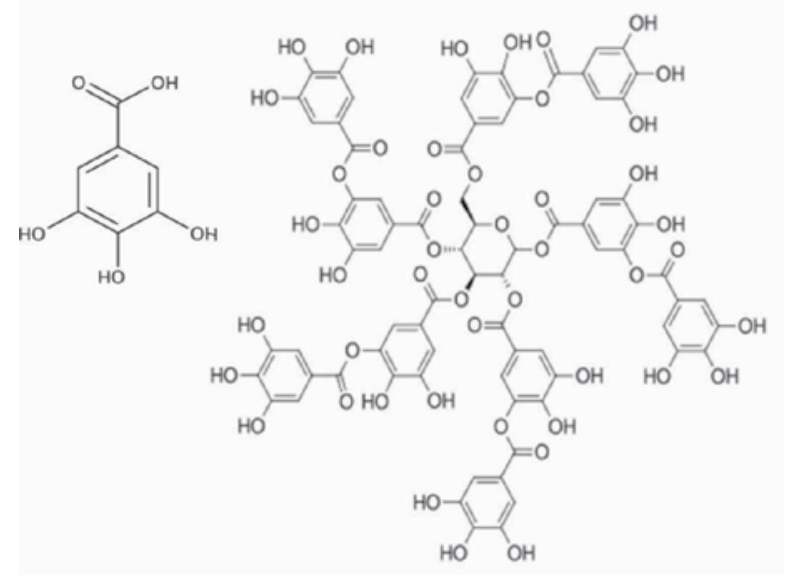

Figure 1. Gallic acid and tannic acid 
mainly in vacuoles and in epidermal and subepidermal layer of leaves and fruit. Their richest source is the bark of various trees, especially very hard South American wood quebracho or acacia wood. A higher content of condensed tannins in dark fruits, vegetables (red beans, cocoa beans, blue grapes) relates to the content of anthocyanins, which are also substances of flavonoid nature and have similar synthesis, as well as in seeds, where they are incorporated together with flavonoids into a comprehensive polymer in the ovary that protects the embryo of the plants from drying [1].

\subsection{Carboxymethyl cellulose (CMC)}

Carboxymethyl cellulose is a cellulose derivative whose skeleton consists of glucopyranose polymer, often used as the sodium salt (Figure 3 ). Some of its hydroxyls are substituted by carboxymethyl groups. CMC is widely used in many industries, especially as a thickener (viscosity change), stabilizer and emulsifier. It is water-soluble, nontoxic, hypoallergenic and shows high swelling. This ballast aditivive is known in the food industry as E466 and it is added e.g. to ice cream, beverages and spreads. CMC stabilizes in acidic dairy products' milk proteins during pasteurization. In addition to food products CMC is also included in cosmetics, eye drops, lubricants, tablets, coatings etc. The interactions of CMC and polyphenols are currently under study such as the actual research of biogenic activity of polyphenols [2]. It is for example shown that CMC has from all the food industry tested polysaccharides the highest ability to mask the bitter taste of polyphenols and tannins in beverages enriched with antioxidants so that it reduces their astringent effect on the salivary glands $[3,4]$. CMC is used to stabilize wine by preventing the precipitation of pottasium bitartrate (tartar) and to prevent the formation of sediment in bottled wines [5]. CMC can also be used as a protective layer for encapsulation of polyphenols for oral use as CMC housing protects these substances against the effects of digestive enzymes, and in addition these substances are safely transported to the place of their maximum resorption in the colon. Only here is the CMC pouch disrupted by the activities of the cellulase enzymes present in intestinal bacteria. CMC is also used as a carrier for active substances and drugs for surface application. Moreover, CMC is part of dressings for treating particular kinds of wound including a mixture of hydrogels and different substances for the stimulation of wound healing. These substances include enzymatic agents, activated carbon, silver ions or antibiotics [6].

\section{Materials and Methods}

\section{$\underline{\text { 2.1 Materials }}$}

Blue grape pomace of Fratava variety (Lobkowicz castle winery Roudnice nad Labem, Ltd.)

Gallic acid (monohydrate) (Sigma-Aldrich)

Powdered sodium carboxymethyl cellulose, medium viscosity, molecular weight 250000 (Fluka)

Folin-Ciocalteau reagent (Penta Chrudim)

Anhydrous sodium carbonate (Lachema) and $\mathrm{NaCl}$ p.a. (Lach-Ner)

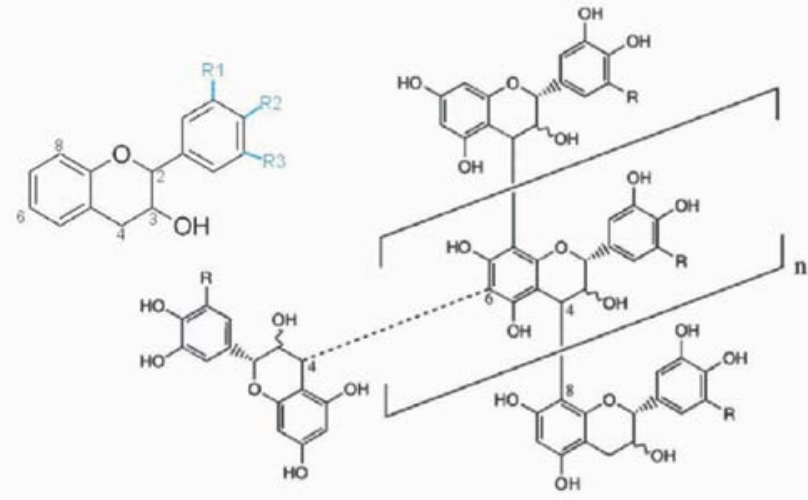

Figure 2. Flavan-3-ol and condensed tannin.

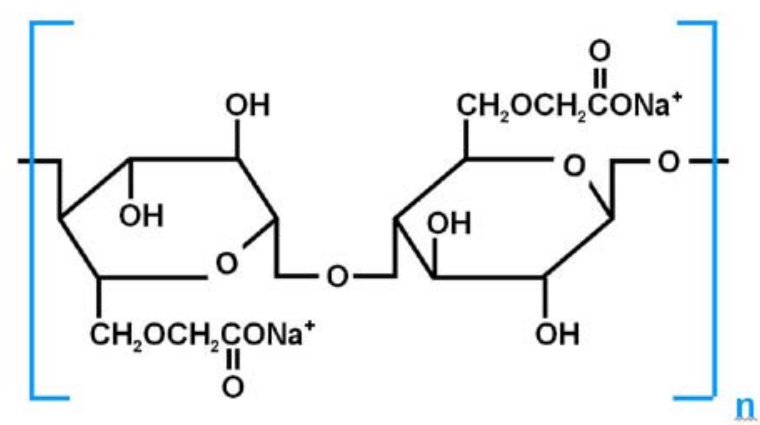

Figure 3. Carboxymethyl cellulose.

\section{$\underline{2.2 \text { Used methods }}$}

\subsubsection{Extraction of polyphenols}

Pomace of blue grape vine were after the pressing of macerated and fermented mash immediately freezed, then dried at $70^{\circ} \mathrm{C}$ to a constant weight, and eventually homogenized. $2 \mathrm{~g}$ of crushed dried pomace (mixed peels, seeds and stems with the weight ratio 15:9:1) were extracted (in a dye cartridge of dyeing apparatus Ahiba Nuance ECO) with $100 \mathrm{ml}$ of distilled water at $100^{\circ} \mathrm{C}$ for 90 minutes.

\subsubsection{Preparation of CMC gels and films}

$3 \mathrm{~g}$ of CMC were dissolved in the extract of pomace by formation of gel. The gel was homogenized, spilled onto glass plate and dried at $60^{\circ} \mathrm{C}$ to film of constant weight. Subsequently, one third of the film was crosslinked at $180^{\circ} \mathrm{C}$ for 1 minute, one third was crosslinked at $180^{\circ} \mathrm{C}$ for 3 minutes to achieve the partial insolubility and one third stayed non-crosslinked [7].

\subsubsection{Determination of total polyphenols}

The total polyphenol content was measured spectrophotometrically using the Folin-Ciocalteu reagent. This reaction is based on colorimetric redox reaction of phenols [8]. To $1 \mathrm{ml}$ of distilled water and $1 \mathrm{ml}$ of Folin-Ciocalteu reagent (diluted 1:9 with distilled water) was added $200 \mu \mathrm{l}$ of skimmed sample (60 revolutions/minute for 3 minutes). $1 \mathrm{ml} 0.75 \mathrm{M}$ solution of anhydrous sodium carbonate was added after 5 minutes. Parallelly, a control sample (a blank one) was also prepared containing $200 \mu \mathrm{l}$ of distilled water, 
and calibration series of solutions of gallic acid (GA) with increasing concentrations from 0.01 to $0.06 \mathrm{mg} / \mathrm{ml}$ (Figure 4). The presence of polyphenols causes after 50 minutes a chemical reaction accompanied by a visual color change of the solution from yellow to blue. Afterwards, the absorbance was measured by the means of UV/VIS spectrophotometer (Helios epsilon) in the absorption maximum at $765 \mathrm{~nm}$. The resulting concentrations of samples (extracts prior to sorption into CMC gel and after the desorption of (MC) were calculated on the basis of calibration curve of gallic acid and expressed in $\mathrm{mg}$ $\mathrm{GA} / \mathrm{ml}$ of solution, respectively in $\mathrm{g} / \mathrm{liter}$.

\subsubsection{Desorption of polyphenols}

$0.5 \mathrm{~g}$ of each of CMC/PF film was immersed in $80 \mathrm{ml}$ saline $(0.9 \% \mathrm{NaCl})$ and dissolved using a magnetic stirrer at $37^{\circ} \mathrm{C}$. A sample was collected each time for the spectrophotometric determination of polyphenols (at intervals $5,10,15,30,45$ and 60 minutes) to ascertain the kinetics of release of polyphenols from CMC/PF gels. The process simulates the contact with the tissues and body fluids such as the course of wound healing.

\section{Experimental Details}

\subsection{Content of PF}

The measured content of polyphenols in the extract was about $0,5 \mathrm{~g}$ of PF/liter (respectively gallic acid equivalent), which is $2,5 \%$ of the weight of the dried pomace. The theoretical content in $100 \mathrm{ml}$ of extract would be therefore $50 \mathrm{mg}$ of PF. After adding of $3 \mathrm{~g}$ of $\mathrm{CMC}$, drying, removing $0,5 \mathrm{~g}$ sample and dissolving in $80 \mathrm{ml}$ of saline, each solution should include ideally about $0,1 \mathrm{mg}$ of $\mathrm{PF} / \mathrm{ml}$ of saline. However, it is necessary to take into account that the CMC hygroscopic powder contained $5 \mathrm{wt}$ \% water and in $100 \mathrm{ml}$ of extract was present (in addition to $50 \mathrm{mg}$ of PF) about $0,5 \mathrm{~g}$ of the dry matter (e.g. dissolved sugars and minerals), and the real content of PF must be less than the theoretical one (Table 1).

\subsection{Release of PF from CMC/PF films}

Figure 5 shows the release of PF from CMC/PF films in saline at $37^{\circ} \mathrm{C}$ using the magnetic stirrer, for 1 hour. The increasing content of PF was measured in the centrifuged samples which were collected at intervals of $5,10,15,30,45$ and 60 minutes. All samples were measured in triplicate and the averages were calculated.

Figure 6 indicates that with the increasing degree of crosslinking of $\mathrm{CMC}$, the half-time release of polyphenols from $\mathrm{CMC}$ film decreases. This leads to two outcomes: first, a thermal crosslinking of CMC occurs resulting in less swelling of CMC. This shortens the diffusion path of substances' molecules which are in the closed path (CMC is inert and does not react chemically with PF - it is only a mechanical sorption) and PF can thus quickly desorb to the place of lower concentration). Second, it is expected that a thermal hydrolysis of polymeric polyphenols (tannins) occur so that smaller PF molecules can again rapidly diffuse from the gel to the solution. The problem is a total thermolability of PF, wherein at $180^{\circ} \mathrm{C}$ their content is declining rapidly; that is why it is important to choose a timetemperature compromise, where their content is still maintained and at the same time their optimum release is under way.

Figure 7 shows a percentage expression of decrease of polyphenols and also percentage expression of decrease of half-time speed of their release from $\mathrm{CMC}$, depending on the

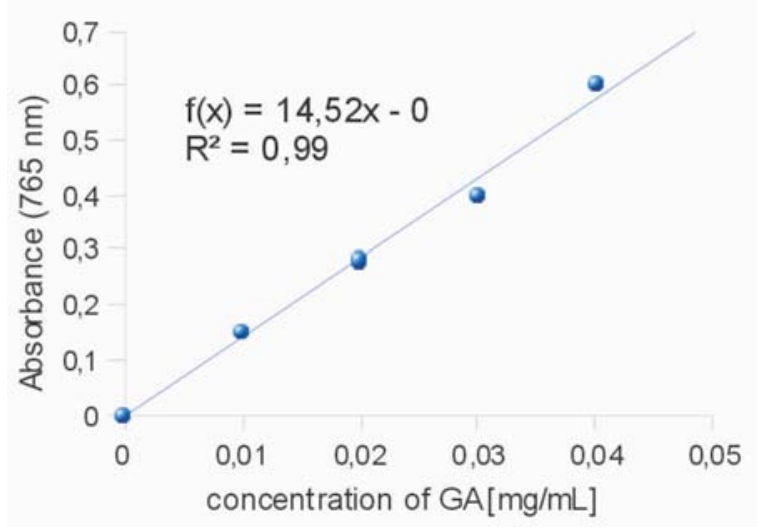

Figure 4. Spectrophotometric calibration of gallic acid.

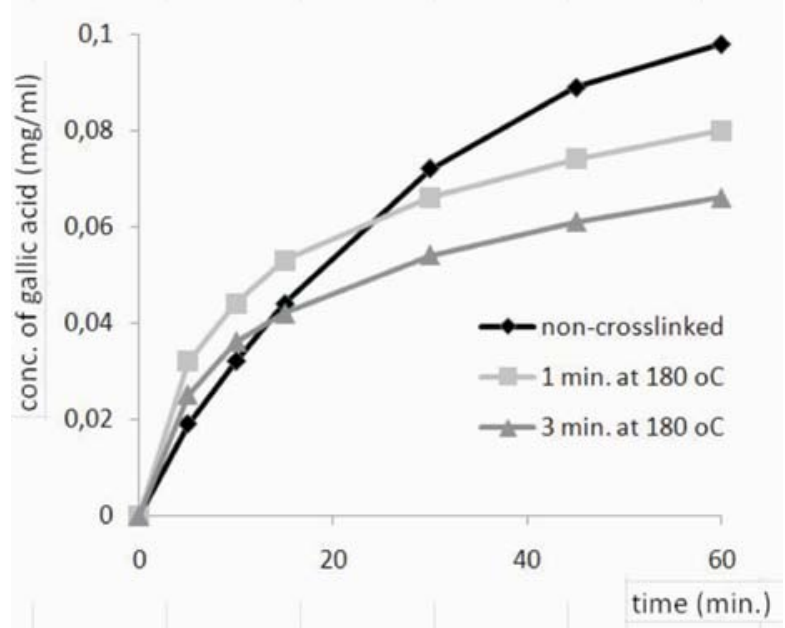

Figure 5. Release of PF from CMC films: non-crosslinked and partially thermal crosslinked CMC

Table 1. PF content in the samples due to the dry matter.

\begin{tabular}{|c|c|c|c|}
\hline Content of CMC & $\begin{array}{c}\text { Total dry matter (dry } \\
\text { matter+CMC+PF) }\end{array}$ & $\begin{array}{c}\text { Content of PF in } \mathbf{0 , 5} \mathbf{g} \\
\text { samples }\end{array}$ & $\begin{array}{c}\text { PF concentration of the } \\
\text { resulting } \mathbf{8 0} \text { ml saline }\end{array}$ \\
\hline $3 \mathrm{~g}$ & $3,40 \mathrm{~g}$ & $7,35 \mathrm{mg}$ & $0,092 \mathrm{mg} / \mathrm{ml}$ \\
\hline
\end{tabular}




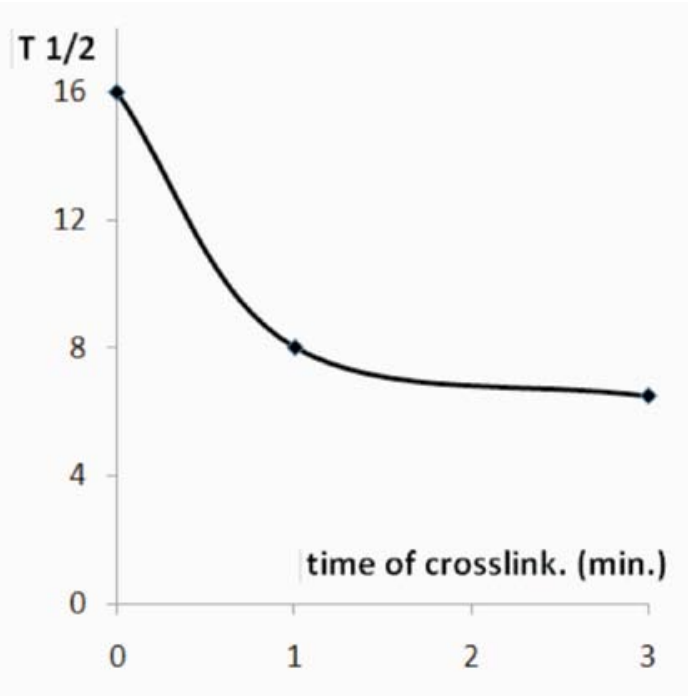

Figure 6. Half time of release of PF from $\mathrm{CMC}$, depending of the degree of crosslinking.

time of thermal crosslinking. It is obvious that, for example, a distance between the two curves is the largest after $1 \mathrm{~min}$. of crosslinking. At this point the release of PF is already short enough while maintaining a high content of PF.

\section{Conclusion}

This research was aimed at the kinetics of release of polyphenols from carboxymethyl cellulose film which was thermal crosslinked at $180^{\circ} \mathrm{C}$ for 1 and 3 minutes and the results were compared with the completely soluble noncrosslinked CMC. Thermolabile PF are partially protected by $\mathrm{CMC}$ and the short high temperatures decreased their content to $85 \%$ of the initial content (and to $70 \%$ respectively). After one hour in saline, the CMC/PF gels were dissolved in 92, 36 and $24 \%$. Depending on the degree of crosslinking, the rate of release increased because partial crosslinking of $\mathrm{CMC}$ reduces its swelling, reducing the diffusion path.

Measurement results show that CMC can be used as a carrier of polyphenols and their release can be influenced by degree of crosslinking and solubility of CMC.

\section{Acknowledgment}

The paper has been supported by the grant project SGS 48008 (provided by Faculty of Textile of Technical University of Liberec) and TACR program ALFA TA01010244 (Czech republic).

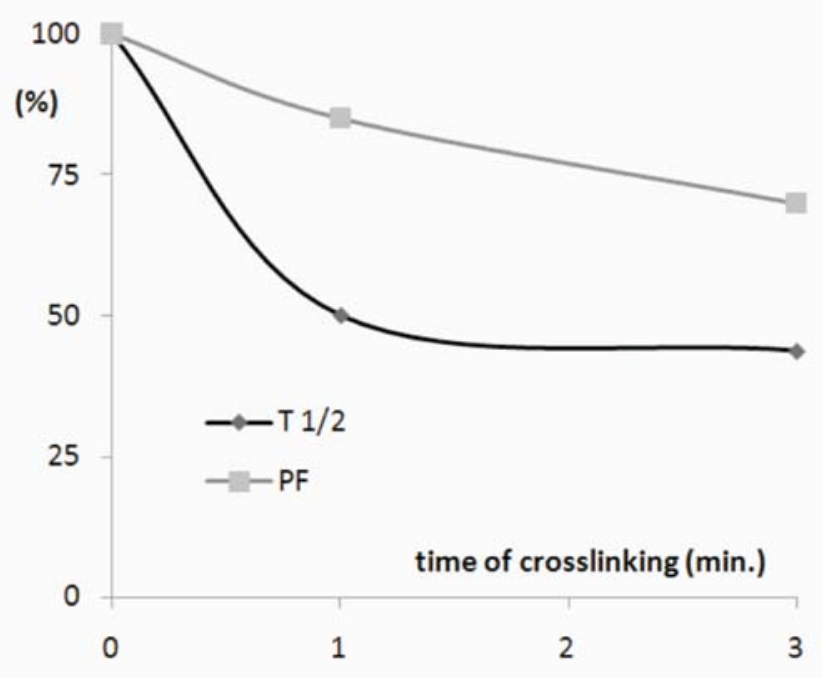

Figure 7. Decrease of PF content and of their half-time release depending time of crosslinking at $180^{\circ} \mathrm{C}$.

\section{References}

[1] Schofield, P., Mbugua, D.M., Pell, A.N.: Analysis of condensed tannins: a review. Animal Feed Science and Technology 91 (2001), p.21-40

[2] Serrano-Cruz, M.R. et al.: Controlled release and antioxidant activity of Roselle (Hibiscus sabdariffa L.) extract encapsulated in mixtures of carboxymethyl cellulose, whey protein, and pectin. Food Science and Technology 50/2 (2013), p. 554-561

[3] Troszyńska,A. et al.:The effect of polysaccharides on the astringency induced by phenolic compounds. Food Quality and Preference 21 (2010), p.463-469

[4] Smith A., K., June, H., Noble, A.C.: Effects of viscosity on the bitterness and astringency of grape seed tannin. Food Quality and Preference 7 (1996), p.161-166

[5] Bosso, A. et al.:Carboxymethylcellulose for the tartaric stabilization of white wines, in comparison with other oenological additives. Vitis 49/2 (2010), p.95-99

[6] Skórkowska-Telichowska, K. et al.: The local treatment and available dressings designed for chronic wounds. Journal of the American Academy of Dermatology (2011), In press

[7] Borůvková, K., Wiener, J., Kukreja, S.: Thermal self crosslinking of carboxymethylcellulose. ACC Journal XVIII/1 (2012), pp. 6-13

[8] Singleton V.L., Orthofer R., Lamuela-Raventós R.M.: Analysis of total phenols and other oxidation substrates and antioxidants by means of folin-ciocalteu reagent. Methods in Enzymology 299, 1999, p.152-178 